\title{
A simple system for the remote detection and analysis of sniffing in explosives detection dogs
}

\author{
IRIT GAZIT \\ Tel-Aviv University, Tel-Aviv, Israel \\ YIZHAR LAVNER \\ Technion IIT, Haifa, Israel \\ and Tel-Hai Academic College, Upper Galilee, Israel \\ GIL BLOCH and OPHIR AZULAI \\ Technion IIT, Haifa, Israel \\ and \\ ALLEN GOLDBLATT and JOSEPH TERKEL \\ Tel-Aviv University, Tel-Aviv, Israel
}

\begin{abstract}
In this article, we describe a device that we have developed, for both research and operational purposes, to assist handlers of sniffer dogs and improve efficiency of search. The device transmits sounds from the dog's muzzle area to a listener, enabling determination of whether the dog is actually sniffing during a search. Both the aural and the computerized sound analyses enable study of the dog's sniffing behavior under a variety of situations. Correlation of these data with the visually monitored behavior of the working dog contributes additional and important information and leads to a greater understanding of detector dogs' behavior and abilities.
\end{abstract}

The earliest known use of dogs as chemical detectors dates back to their operation as hunting dogs 12,000 years ago, as based on tomb evidence (Furton \& Myers, 2001). Since World War II, dog-handler teams have been used extensively by the military to locate explosives. Over the last century, the use of detector dogs has expanded and is currently widespread throughout the world. Detector dogs primarily rely on their sense of smell to detect a variety of odors for military and civilian uses. Military working dogs are used for the detection of explosives, land mines, trip-wires, weapons, and so forth. (for a comprehensive review, see Frost, 1990). The civilian uses are manifold, such as searching for live or dead bodies in open field areas (Komar, 1999) or for survivors buried beneath rubble at disaster areas, crossmatching human odors (Schoon, 1996, 1998; Schoon \& De Bruin, 1994), and investigating arson (Gialamas, 1996; Kurz et al., 1994), as well as detecting smuggled agricultural products, drugs, gaspipe leaks, cows in estrus (Hawk, Conley, \& Kiddy, 1984; Jezierski, 1992), melanomas (Williams \& Pembroke, 1989), termites,

We gratefully acknowledge N. Paz for her editorial assistance. We also thank the dog handlers and trainers and the dogs themselves for participating in this study. Correspondence concerning this article should be addressed to I. Gazit, Department of Zoology, George S. Wise Faculty of Life Science, Tel-Aviv University, Tel-Aviv 69978, Israel (e-mail: iritgazi@ post.tau.ac.il). screwworms (Welch, 1990), and so forth. Their ability to detect the revealing odors derives from the dogs' possession of a very large number of olfactory receptor cells, together with a specialized behavior called sniffing.

In both humans and other mammals, perception of an odor in the environment usually initiates a sniffing episode composed of several sniffs. It is generally accepted that this behavior enhances odor perception (Laing, 1983). Other studies have shown that larger than normal flow rates of air through the nose enhance odor perception (Le Magnen, 1945; Rehn, 1978). Neuhaus (1981) verified that during normal breathing of the dog, the pressure differences between inspiration and expiration in the rear region of the nasal space are too low to transport odor molecules to the remote part of the olfactory mucosa. However, molecules are able to diffuse effectively into these remote areas when high odor concentrations and a minimal sniff inspiration time of $2 \mathrm{sec}$ are maintained. As a result of these pressure differences in the nasal passages, at the end of the inspiration puff an odor will reach those parts of the olfactory epithelium in a remote location from the breathing flow. Steen, Mohus, Kvesetberg, and Walloe (1996) showed that while searching for ground scent, their dogs sniffed at a frequency of up to 200 times per minute, a strategy that may create turbulence in the nasal passages and thereby enhances transport of scent molecules to the receptors in the ethmoidal cavity. Sobel et al. (1998), on the basis of Le Magnen (1945) and Laing 
(1983), emphasized the importance of sniffing to olfaction: "The sensation and perception of smell are largely dependent on sniffing, which is an active stage of stimulus transport and therefore an integral component of mammalian olfaction" (p. 282). Sniffing induces oscillation in the olfactory bulb, driving the piriform cortex in the temporal lobe at the frequency of sniffing (Adrian, 1942; Bressler, 1987; Bressler \& Freeman, 1980; Ueki \& Domino, 1961).

Despite the well-recognized importance of sniffing for the detection and identification of odors, on the one hand, and the extensive use of detector dogs around the world, on the other, there have been few studies in which the variables affecting sniffing behavior in dogs have been investigated. Consequently, the influences of a variety of situations on the dogs' detection abilities are barely known.

While searching for the target odor, the dog performs sniffing in order to bring the odor into the nose, where it comes into contact with the olfactory receptors. During sniffing, air is inhaled through the nostrils in short aspirations while the mouth remains closed. Because dogs do not possess sweat glands, panting is the main means of cooling the body. Panting constitutes rapid respiration in order to regulate body temperature by increasing the vapor rate from passage of respiration (Crawford, 1962). During panting, most of the air passes through the mouth (Schmidt-Nielsen, Bretz, \& Taylor, 1970). Since the dogs can thus either sniff or pant but can never perform both actions simultaneously, panting will consequently cause a decrease in sniffing rate.

Despite the wide variety of possible uses for the olfactory ability of dogs, owing to the international problem of terrorism most of these working dogs around the world are used for explosives detection. Explosives detector dogs are used both on and off leash. The advantages of on-leash dogs are that the handler can closely observe the behavior of the dog and determine whether or not it is sniffing, as well as ensure that the dog searches all relevant areas. The disadvantages of working with onleash dogs are that it is potentially dangerous to the handler and also inhibits the dog from searching at its optimal rate. In addition, in many situations, owing to security or other considerations for which the handler has to remain at a safe distance from the working dog, he or she cannot know whether the dog has performed a completely thorough search. A similar situation occurs when working in darkness, bushy areas, crowded hangars, and so on, where it is impossible to remain in constant visual observation of the dog's behavior and nose work.

For such situations, we have developed a simple wireless device that is worn by the off-leash working dog and lets the handler know whether the dog is sniffing sufficiently as it moves through the area. This feedback from the wireless unit allows the handler to make an informed decision about directing the dog to re-search specific areas.

The apparatus consists of a wireless omnidirectional microphone-AT803b, manufactured by Audio-Technica
(Audio-Technica U.S., Inc., 1221 Commerce Drive, Stow, $\mathrm{OH} 44224)$ and a transmitter (Tx2000, Audio-Technica) attached to a specially designed muzzle worn by the dog (see Figure 1). The handler, who is following the dog from a distance, is able to hear the sounds originating from the area of the nose and mouth, including sniffing and panting, which are transmitted to his radio receiver (Mx2000, Audio-Technica) and headphones. The radio signal can also be transmitted to the audio input of a video camera so that any visual recording of the dog's behavior can be later analyzed and correlated with the sound recording. For detailed unit specifications, see the Appendix.

The specially designed muzzle is fitted to the dog with the attached wireless microphone close to the nostrils (see Figure 1). The muzzle comprises a soft flexible strap that wraps around the dog's mouth and nose (the upper part consists of a Velcro strap to allow close adjustment to dog's head; the lower part consists of a flexible rubber strap on each side to keep the muzzle firmly in place against the throat). The muzzle has been designed to allow the dog to pant freely, as well as to eat while the muzzle is in place. The microphone is firmly attached to the muzzle by Velcro strips above the dog's nose (about 1-3 cm from the nostrils), without interfering with its field of vision, and enables efficient recording of the sniffing and panting performed by the dog during working. The microphone remains immobile against the dog's nose even when the dog scratches its nose, rubs against bushes, and so on. The use of flexible straps and Velcro allows the muzzle to be fitted individually to any medium-sized dog.

The transmitter can be conveniently attached to the dog's collar or harness as required. It is possible to utilize the normal harness used with police or search and rescue dogs. The wire connecting the microphone to the transmitter is without slack in order to avoid entangling in the dog's legs or in bushes.

Dogs that are accustomed to an ordinary muzzle usually adapt to this muzzle in 3-4 days and work without

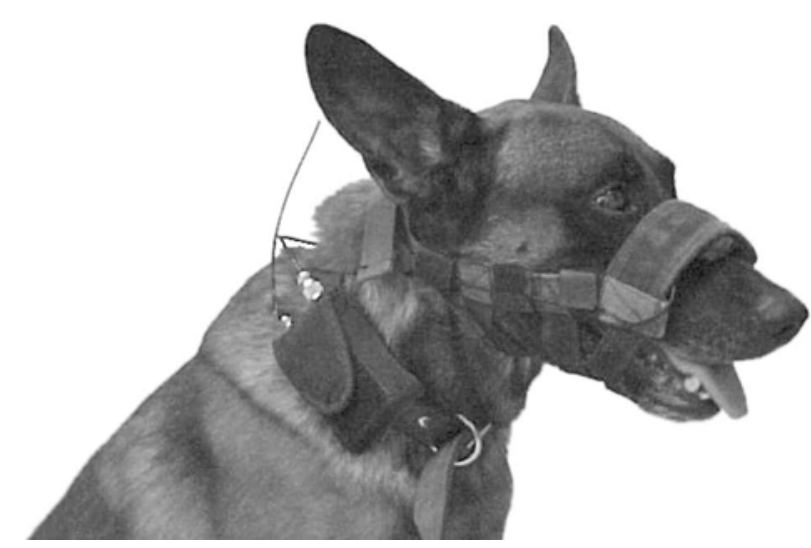

Figure 1. Dog wearing the apparatus (photographed by I.G.). 
appearing to be bothered by it. Indeed, all our dogs swiftly acclimated to the equipment. A microphone attached to a dog's head in order to record and analyze sniffing was first used by Thesen, Steen, and Doving (1993). However, since no muzzle was used to firmly anchor the microphone above the dog's nose, their system is inappropriate for use in heavy vegetation, as opposed to open areas. Moreover, Thesen and his colleagues did not specify the characteristics of their equipment, since they took advantage of specialized military components. The muzzle that we have developed and used in the present study enables work in a variety of surroundings (e.g., grassy or bushy areas) and can be adapted for use by all dogs, after brief habituation. The system described here is constructed from commercially available off-the-shelf components.

This system enables the listener to easily discriminate between sniffing and nonsniffing or panting. The uniqueness of the apparatus lies in its ability to monitor a dog's behavior from a great distance (up to $100 \mathrm{~m}$ in an open wooded area), thus allowing the handler to determine whether or not the dog is sniffing while moving through a particular area. This in turn allows an informed decision about directing the dog to re-search specific areas. The system was tested and found to be reliable in three conditions: an open wooded area, an open field, and inside buildings. In addition, the system can also increase the efficiency with which dogs can be trained in odor detection for a variety of goals. The basic principles of con- ditioning are used throughout all phases of dog training, which is continuous throughout the dog's career, in order to maintain proficiency (Technical Training, 1996). Since most training techniques are based on both classical and operant conditioning principles, reinforcement should be given immediately following the correct response. Using the system enables the handler to unmistakably identify the correct response of the dog, thus both avoiding incorrect reinforcement and enabling perfect timing for correct reinforcement. We believe that this device has great potential for both operations and research. Owing to the high reliability of performance of this unit and its effective use under all field conditions tested so far without interference from the surroundings in which the dog is searching, the device is also highly suitable for research purposes.

If the device is to be used for research purposes, the recorded signals can be analyzed later by using a special computer software program that discriminates between sniffing and panting and counts the signals according to the experimenter's requirements. For this purpose, a computerized system for automatic detection and discrimination of sniffing and panting events was developed. The main program is constructed in the Matlab environment, using the neural networks module (Version 5.3) on a personal computer. The input to the system is the acoustic signal, sampled at a $44100 \mathrm{~Hz}$ and downsampled to $11025 \mathrm{~Hz}$ (Figure 2). The output of the system is a se-

(A) Panting samples at $44200 \mathrm{~Hz}$.

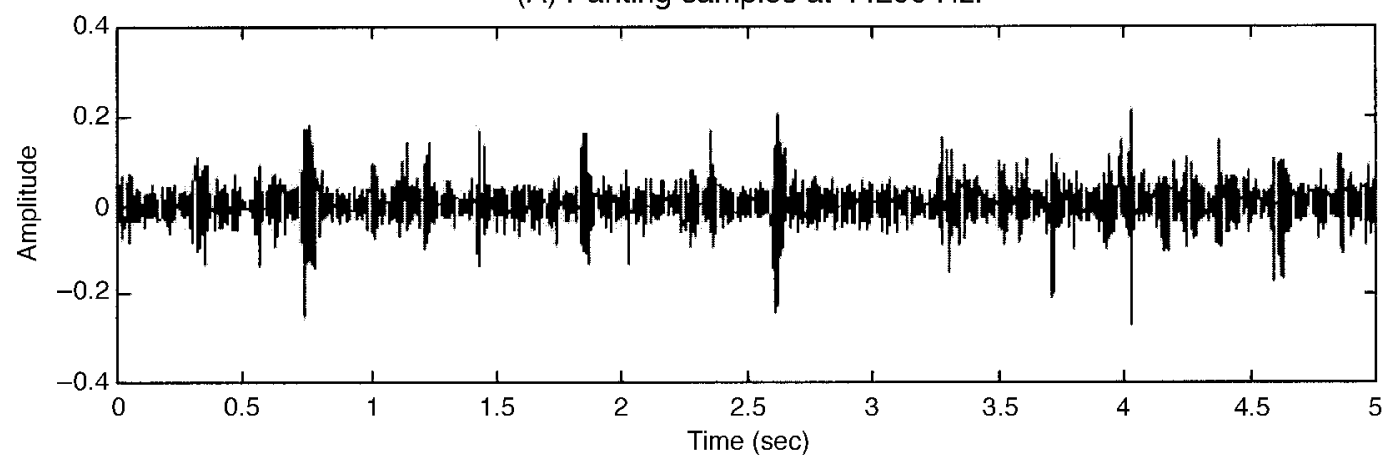

(B) Sniffing samples at $44200 \mathrm{~Hz}$.

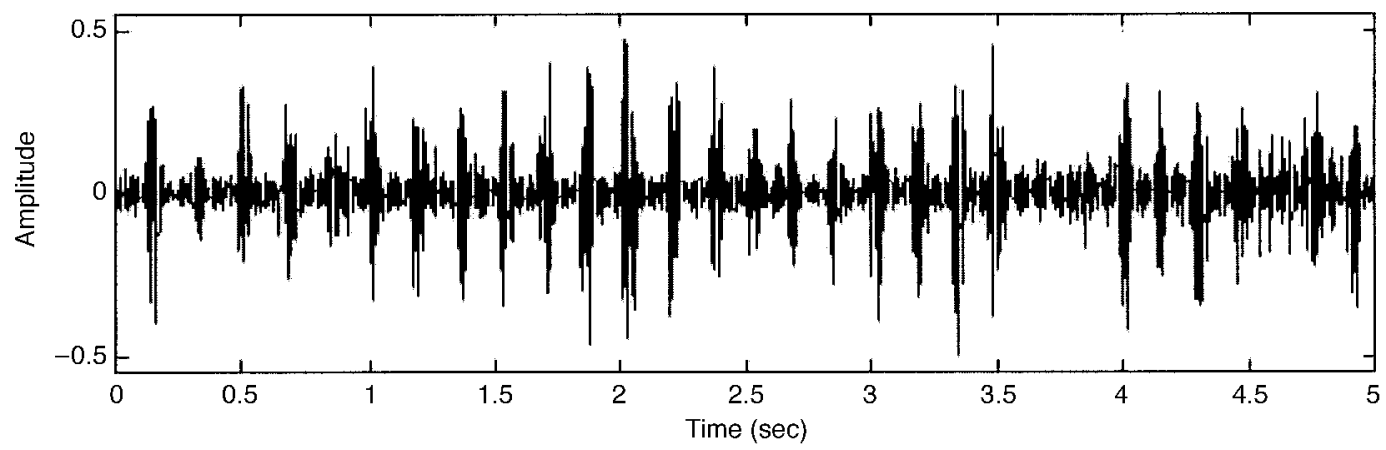

Figure 2. Time waveform of the sniffing and panting sounds. (A) Panting waveform. (B) Sniffing waveform. Both were sampled at $44200 \mathrm{~Hz}$. 
quence of marks along the time waveform, indicating the beginning and end of each event and whether it is sniffing or panting.

Before the algorithm was applied, each sound was classified by the first author (I.G.) as a sniff or a pant on the basis of the auditory cues. An intensive signal analysis was carried out in order to characterize the sniffing and the panting signals. Various features and parameters were extracted and tested as candidates for the detection and discrimination processes. Both time-domain and frequency-domain features were studied. In the time domain, the short-time energy function, the autocorrelation function, and the zero-crossing rate were calculated for each event. The events (whether sniffing or panting) were then demarcated for further analysis, using the short-time energy function. Those sections of the signal that contained silence or recording noise were removed. In the frequency domain, the Fourier transform of each event was calculated, and resonance frequencies were detected. An autoregressive (AR) model was utilized for analysis of the signals. The spectral envelope was evaluated using the autocorrelation method of linear prediction (Makhoul, 1975). The spectral envelope of several sniffing and panting events can be seen in Figure 3. Parameters in the joint time-frequency domain were also examined using the short-time Fourier transform (Oppenheim \& Schafer, 1999), which shows features that do not appear in any of the domains separately. Since timedomain parameters did not show significant differences between sniffing and panting sounds, we focused on the frequency domain. Several parameters derived from the spectral envelope were selected for the discrimination process.

The features were selected according to their discriminatory power. The features were examined in three orders of the AR model (3, 10, and 20). Four amplitude values of frequencies in the spectral envelope of an AR model of order 20 and four slopes in those curves were found to be most discriminative in 6 dogs (Figure 4). These parameters were the input to the neural network in the discrimination stage.

A three-layer feed-forward neural network was applied for the discrimination. The network was constructed of eight neurons in the input layer, four in the hidden layer, and one in the output layer. A log-sigmoid transfer function was used for the input and hidden neurons, and a linear function was used for the output neuron. A schematic description of the network is shown in Figure 5.

The basic algorithm of the system is depicted in Figure 6. The algorithm is divided into two stages. The first is the learning stage, in which the neural network is trained to discern the two types of events. The neural network is trained, and the weights are set according to labeled sounds. Each learning stage is performed separately for each dog. For each dog, the individual parameters are extracted and used by the neural network. In the second, discrimination phase, the classification into sniffing and panting events is applied, using the trained network. The results of the network output decisions are collected to a database for further analysis and are shown graphically

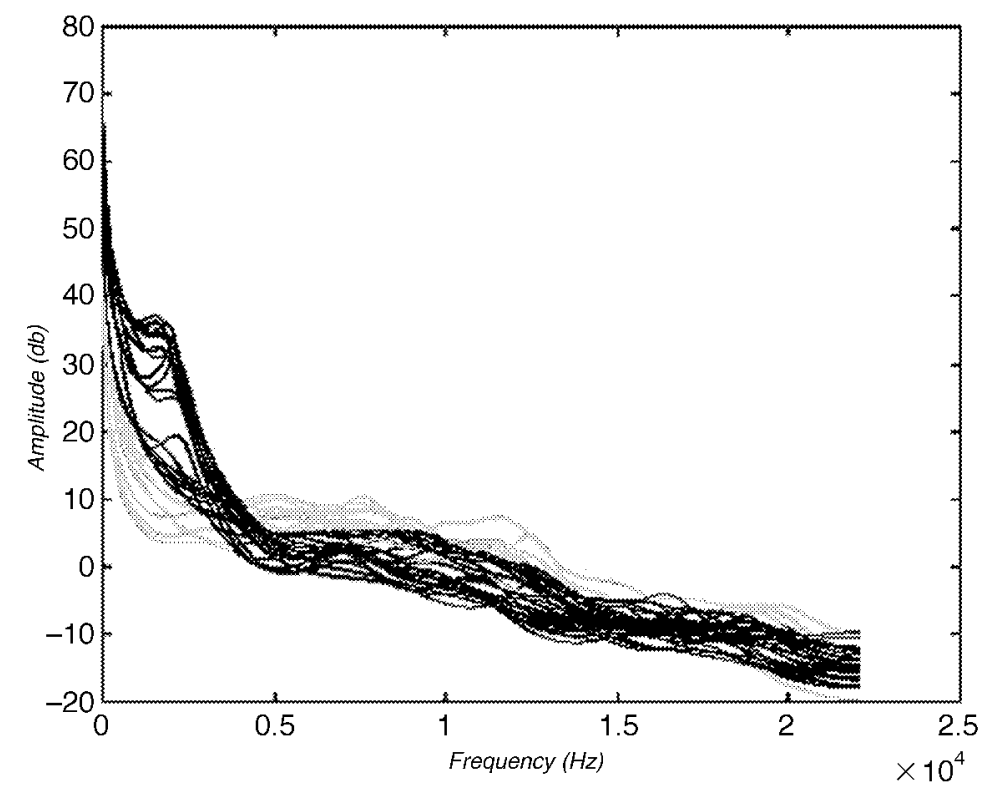

Figure 3. Evaluated spectral envelopes for sniffing and panting signals using the autoregressive (AR) model. The spectral envelopes were calculated using $L P C$ analysis of order 20 on labeled events: sniffing (gray curves) and panting (black curves) events (energy periods only). The distinction between the sniffing and the panting spectral characteristics is easily seen, especially in the lower frequency range. 

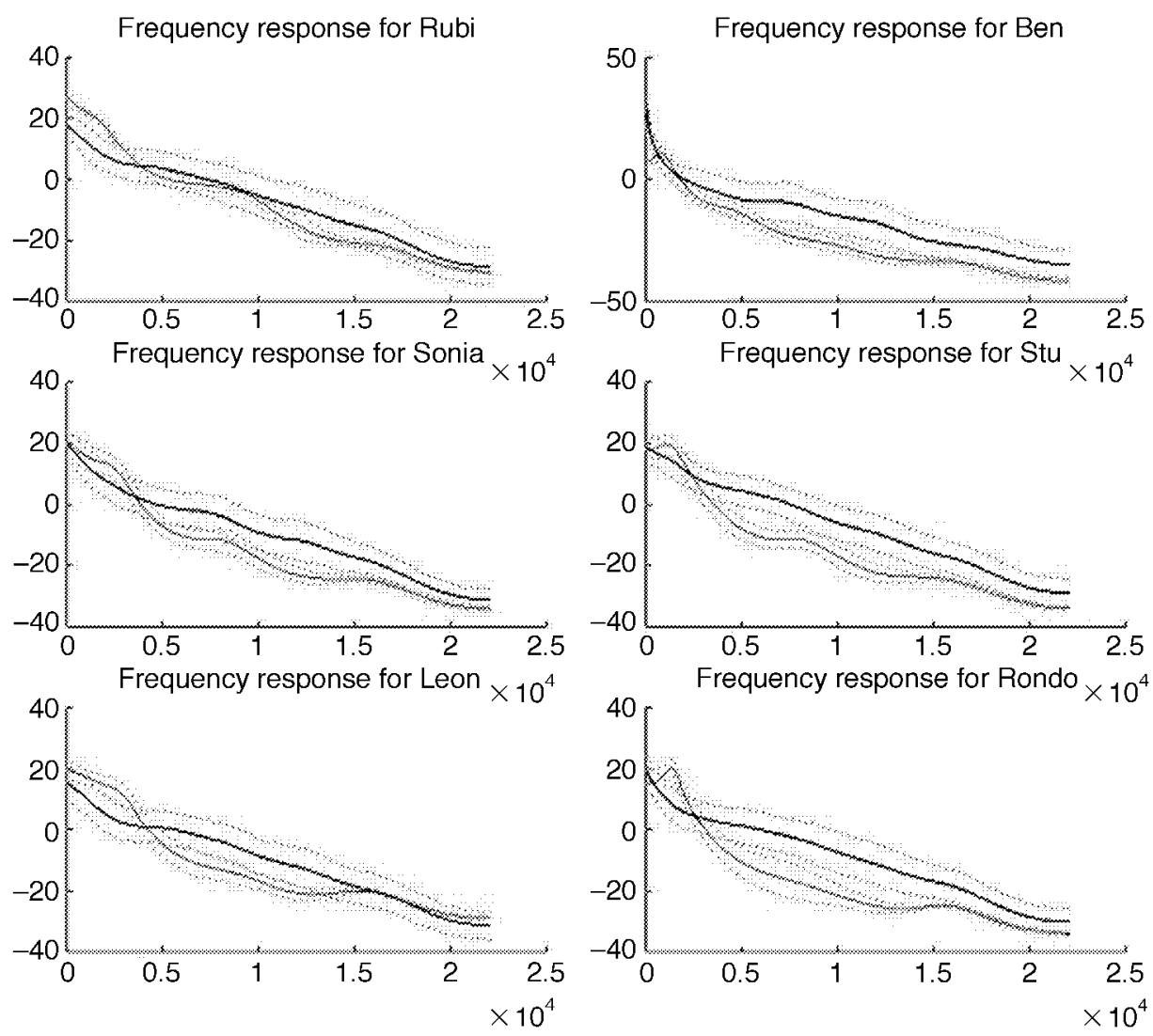

Figure 4. Average frequency responses for different dogs' signals. Each curve (bold line) represents an average spectral envelope, and the dotted lines represent standard deviation curves. The difference between the sniffing and the panting frequency responses was used as the basis for the discriminative features.

in the user interface of the system. A more detailed description of this system will be published elsewhere (submitted to the Journal of Bioacoustics).

In order to evaluate the learning reliability of the system, the recordings of 6 dogs were selected, each of which contained about 120-200 events for sniffing (total number, 939) and about 120-300 for panting (total number, 1,094). After the software had learned the individual characteristics of sniffing for each dog, an analysis was made of another sniffing recording of the same dog. In addition, the first author (I.G.) had classified the same recording into sniffing or panting, independently of the computer classification. A comparison was then made between computer and author classifications in order to assess the precision of the computer classification. The same procedures were performed for panting in order to evaluate the software's reliability of learning the panting signals for each dog. Correct identification rates of up to $91.1 \%$ were achieved from the acoustic signals of 6 dogs for sniffing, and $95.3 \%$ were achieved for panting (see Table 1). In order to evaluate the analysis performance and reliability of the system, 20 recordings of explosives searches by 6 dogs were selected, each of which con- tained about 119-259 events for sniffing (total number of sniffing, 1,073) and about 41-171 for panting (total number, 635). At this stage, after the software had learned the individual characteristics of sniffing and panting for each dog, an analysis was made for each dog's real explosives search. Correct identification rates of $89.8 \%$, on average, for sniffing were achieved from

Table 1

Performance Reliability of the System During the Learning Phase of Sniffing and Panting Events for Each Dog

\begin{tabular}{lccccc}
\hline & \multicolumn{2}{c}{$\begin{array}{c}\text { Performance } \\
\text { Reliability for Sniffing }\end{array}$} & & \multicolumn{2}{c}{$\begin{array}{c}\text { Performance } \\
\text { Reliability for Panting }\end{array}$} \\
\cline { 2 - 3 } \cline { 5 - 6 } Dog's Name & Precision (\%) & No. & & Precision (\%) & No. \\
\hline Ben & 92.4 & 158 & & 86.0 & 122 \\
Leon & 96.3 & 161 & & 98.2 & 119 \\
Rondo & 85.4 & 123 & & 100.0 & 158 \\
Rubi & 84.0 & 200 & & 89.2 & 213 \\
Sonia & 94.0 & 151 & & 98.6 & 291 \\
Stu & 94.5 & 146 & & 99.5 & 191 \\
$\quad$ Mean $\pm S E$ & $91.1 \pm 2.1$ & 939 & & $95.3 \pm 2.5$ & 1,094 \\
\hline
\end{tabular}

Note-Data show degree of precision (\%) of the computerized software for each dog and the number of events. 


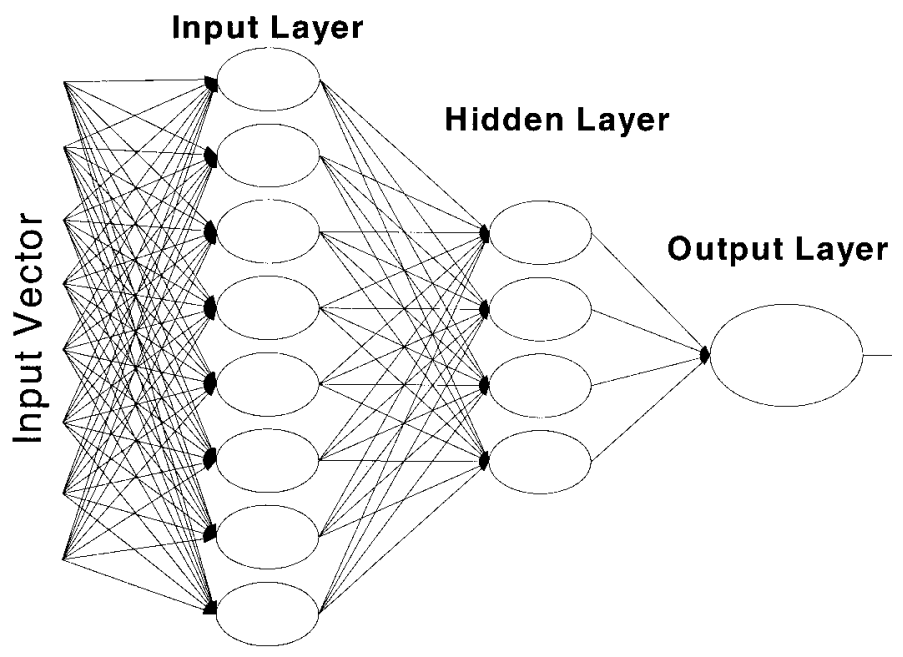

Figure 5. A schematic description of the feed-forward neural network used for the discrimination. The input layer contains eight neurons, the hidden layer four neurons, and the output layer one neuron. In the input and hidden layers, a log-sigmoid transfer function was used, and a linear function was used for the output neuron.

the acoustic signals of 6 dogs acquired from these real explosives search sessions, and an average of $82.8 \%$ was achieved for panting (see Table 2).

To date, the system has been used within an army training framework, aimed at testing the reliability of the dogs' work. On these occasions, we did not collect data systematically, but rather, assessment was based on the handlers' reports. When the level of sniffing was low, the handlers reported a low percentage of success in the finding of targets. In many cases, the handlers then re-sent the dogs back to the same area with the "sniff" command, and success in finding targets increased.

\section{A. Learning Phase}
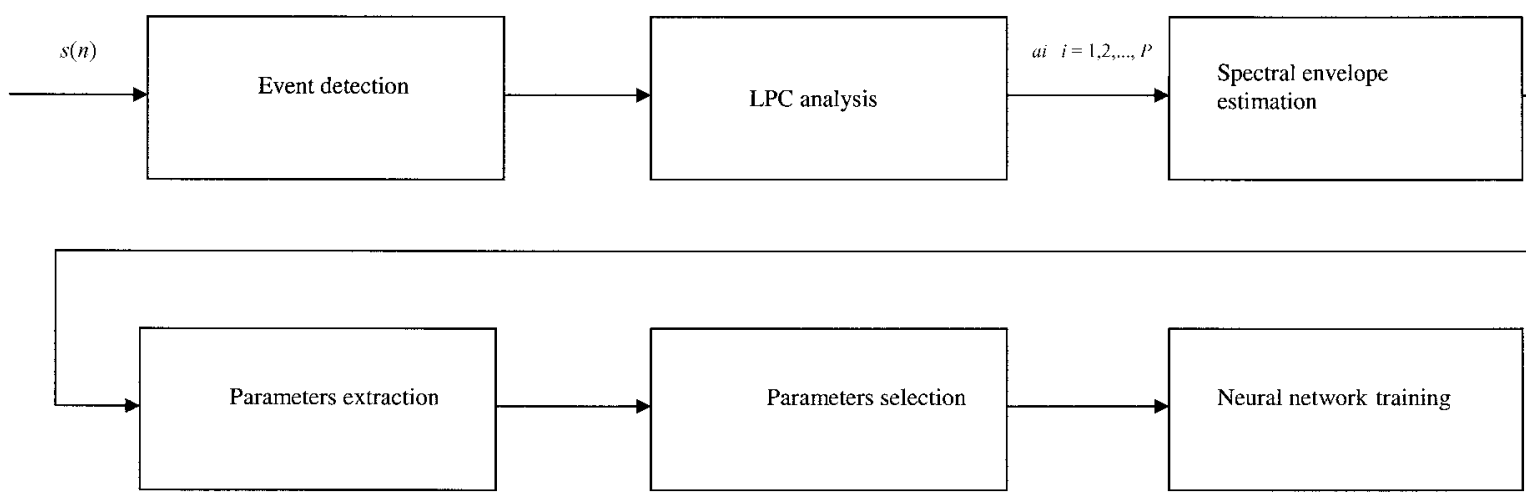

\section{B. Discrimination Phase}

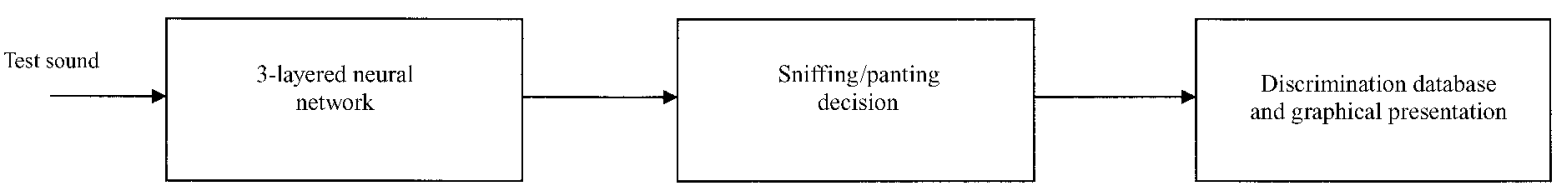

Figure 6. A schematic description of the discrimination algorithm. (A) Learning stage. This includes event detection using a shorttime energy function, the spectral envelope estimation using LPC analysis, and features selection for the discrimination and training of the neural networks. Labeled sniffing and panting events were used for this stage. (B) Discrimination stage, in which test events are detected and discriminated, using the trained neural network. 
Table 2

Performance Reliability of the System for 20 Explosives Search Recordings for 6 Dogs

\begin{tabular}{lccccr}
\hline & \multicolumn{2}{c}{$\begin{array}{c}\text { Performance } \\
\text { Reliability for Sniffing }\end{array}$} & & \multicolumn{2}{c}{$\begin{array}{c}\text { Performance } \\
\text { Reliability for Panting }\end{array}$} \\
\cline { 2 - 3 } Dog's Name & Precision $(\%)$ & No. & & Precision (\%) & No. \\
\hline Ben & 84.1 & 195 & & 82.3 & 85 \\
Leon & 94.7 & 119 & & 85.4 & 41 \\
Rondo & 97.4 & 152 & & 91.7 & 48 \\
Rubi & 96.1 & 259 & & 77.4 & 159 \\
Sonia & 85.0 & 213 & & 74.8 & 131 \\
Stu & 81.5 & 135 & & 85.4 & 171 \\
$\quad$ Mean $\pm S E$ & $89.8 \pm 2.9$ & 1,073 & $82.8 \pm 2.5$ & 635 \\
\hline
\end{tabular}

Note-Data show degree of precision (\%) of the computerized software for each dog and the number of events.

Although, in the course of this study, we examined two breeds of dogs (Belgian malinois and Labrador retriever), it could be interesting to examine this system with other breeds too. However, since sniffing and panting are basic natural behaviors, common to all dogs, we believe that the system will prove equally reliable with other breeds as well.

We believe that this system can be used for research in many other areas, such as assessment of the olfactory functioning of canines under different conditions: for assessment following such diseases as canine parainfluenza viral infection, for assessment of age-related changes in canine olfaction, and as an optional technique for diagnosis of anosmia in dogs. It can also be used to assess a dog's functional ability under different environmental conditions (e.g., bad weather, darkness, etc.) or "psychological" ones, such as the effects of a lack of motivation or growing frustration on the dog's behavior. Use of this system in research can offer an important tool for studying canine olfaction, as well as its obvious contribution to improving operational performances.

\section{REFERENCES}

Adrian, E. D. (1942). Olfactory reactions in the brain of the hedgehog. Journal of Physiology, 100, 459-473.

Br essl er, S. L. (1987). Relation of olfactory bulb and cortex: II. Model for driving of cortex by bulb. Brain Research, 409, 294-301.

Bressl er, S. L., \& Freeman, W. J. (1980). Frequency analysis of olfactory system EEG in cat, rabbit and rat. Electroencephalography \& Clinical Neurophysiology, 50, 19-24.

Crawf or d, E. C. (1962). Mechanical aspects of panting in dogs. Journal of Applied Physiology, 17, 249-251.

Frost, D. F. (1990). A centralized source of information for the military working dog program. Unpublished master's thesis. U. S. Army Command and General Staff College, Fort Leavenworth, KS.

Furt on, K. G., \& Myers, L. J. (2001). The scientific foundation and efficacy of the use of canines as chemical detectors for explosives. Talanta, 54, 487-500.

Gial a mas, D. M. (1996). Enhancement of fire scene investigations using accelerant detection canines. Science Justice, 36, 51-54.

Hawk, H. W., Conl ey, H. H., \& Kiddy, C. A. (1984). Estrus-related odors in milk detected by trained dogs. Journal of Dairy Science, 67, 392-397.

Jezier ski, T. (1992). The effectiveness of estrus detection in cows by trained dog. Animal Science Papers \& Reports, 10, 57-66.

Komar, D. (1999). The use of cadaver dogs in locating scattered, scavenged human remains: Preliminary field test results. Journal of Forensic Sciences, 44, 405-408.

Kurz, M. E., Bil 1 ard, M., Ret t ig, M., August iniak, J., Lange, J., Lar sen, M., Warrick, R., Mohns, T., Bora, R., \& Broadus, K. (1994). Evaluation of canines for accelerant detection at fire scenes. Journal of Forensic Sciences, 39, 1528-1536.

Laing, D. G. (1983). Natural sniffing gives optimum odour perception for humans. Perception, 12, 99-117.

Le Magnen, J. (1945). Étude des facteurs dynamiques de l'excitation olfactive. L'Année Psychologique, 45-46, 77-89.

Makhou 1, J. (1975). Linear prediction: A tutorial review. Proceedings of the IEEE, 63, 561-580.

Neuhaus, V. W. (1981). The importance of sniffing to the olfaction of the dog. Zeitschrift für Saugetierkunde, 46, 301-310.

Oppen heim, A. V., \& Schafer, R. W. (1999). Discrete-time signal processing (pp. 714-722). Upper Saddle River, NJ: Prentice-Hall.

Reh n, T. (1978). Perceived odor intensity as a function of air flow through the nose. Sensory Processes, 2, 198-205.

Sch midt -Niel sen, K., Bret z, W. L., \& Tayl or, C. R. (1970). Panting in dogs: Unidirectional air flow over evaporative surfaces. Science, 169, 1102-1104.

Schoon, G. A. A. (1996). Scent identification line-ups by dogs (Canis familiaris) trained in a tube-retrieving method: Experimental design and forensic application. Applied Animal Behaviour Sciences, 49, 257-267.

Schoon, G. A. A. (1998). A first assessment of the reliability of an improved scent identification line-up. Journal of Forensic Sciences, 43, 70-75.

Schoon, G. A. A., \& De Br u in, J. C. (1994). The ability of dogs to recognize and cross-match human odours. Forensic Science International, 69, 111-118.

Sobel , N., Prabhakar an, V., Desmond, J. E., Gl over, G. H., Goode, R. L., Sullivan, E. V., \& Gabriel i, J. D. E. (1998). Sniffing and smelling: Separate subsystems in the human olfactory cortex. Nature, 392, 282-286.

St een, J. B., Mohus, I., Kveset berg, T., \& Wall oe, L. (1996). Olfaction in bird dogs during hunting. Acta Physiologica Scandinavica, 157, 115-119.

TECHNICAL TRAINING. (1996). Military working dog handler (block 1) 37 th training group, 341 st training squadron (Study guide workbook SGWBL3ALR3PO32A-000AI). Lackland Air Force Base, Texas.

Thesen, A., St een, J. B., \& Doving, K. B. (1993). Behavior of dogs during olfactory tracking. Journal of Experimental Biology, 180, 247-251.

Ueki, S., \& Domino, E. F. (1961). Some evidence for a mechanical receptor in olfactory function. Journal of Neurophysiology, 24, 12-25.

Wel ch, J. B. (1990). A detector dog for screwworms (Diptera: Calliphoridae). Journal of Economic Entomology, 83, 1932-1934.

Will iams, H., \& Pembroke, A. (1989). Sniffer dogs in a melanoma clinic? Lancet, 1, 734. 


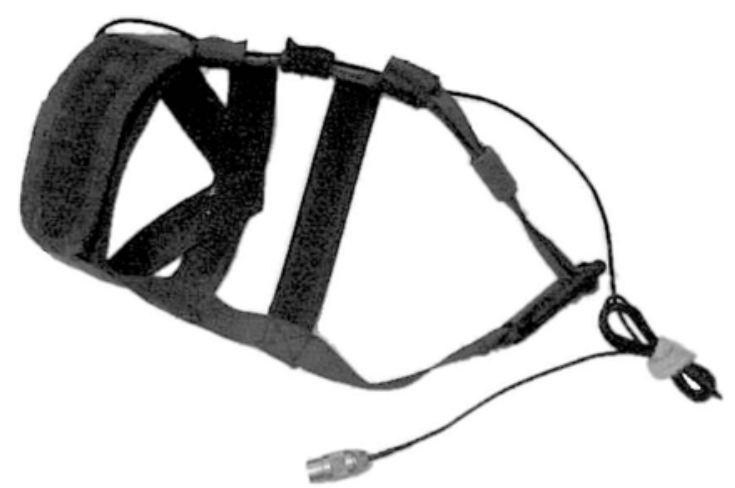

Figure A1. The wireless microphone attaches to the muzzle (photographed by I.G.).

The equipment used in this study was selected to be suited to the study requirements on the basis of operational needs. Alternative equipment and, thus, different specifications are possible; selection has to be made according to one's needs (see Figure A1).

\section{Microphone AT803b Specifications}

Element: Fixed-charge back plate permanently polarized condenser

Frequency response: $30-20,000 \mathrm{~Hz}$

Low frequency roll-off: $150 \mathrm{~Hz}, 6 \mathrm{~dB} /$ octave

Open circuit sensitivity: $-46 \mathrm{~dB}(0.5 \mathrm{mV})$ re $1 \mathrm{~V}$ at $1 \mathrm{~Pa}$

Impedance: $200 \mathrm{ohms}$

Maximum input sound level: $124 \mathrm{~dB}$ SPL, $1 \mathrm{kHz}$ at $1 \%$ T.H.D.

Dynamic range: $95 \mathrm{~dB}, 1 \mathrm{kHz}$ at max SPL

Signal-to-noise ratio: $65 \mathrm{~dB} 1 \mathrm{kHz}$ at 1 pa

Battery type: AA/UM3 $1.5 \mathrm{~V}$

Weight: microphone $2.5 \mathrm{~g}(0.09 \mathrm{oz})$; power module, $147 \mathrm{~g} \mathrm{(5.2 \textrm {oz } )}$

Dimensions: microphone, $20.5 \mathrm{~mm}$ (0.81 in.) long, $10.0 \mathrm{~mm}$ (0.39 in.) diameter; power module, $83.0 \mathrm{~mm}$ (3.27 in.) $\mathrm{H} \times 63.0 \mathrm{~mm}(2.48$ in.) $\mathrm{W} \times 22.0 \mathrm{~mm}(0.87$ in. $) \mathrm{D}$

\section{Transmitter and Receiver Specifications}

S/N ratio: 96 to over $104 \mathrm{~dB}$

Frequency response: $50 \mathrm{~Hz}$ to $15 \mathrm{kHz} \pm 1 \mathrm{~dB}$

THD, 6-dB input as gross overload on max Gain position $7:<0.2 \%$

THD @ working levels: $<0.1 \%$

Battery required: MN1604

\section{Tx2000 Transmitter}

Weight: $105 \mathrm{~g}$

Size: $85 \times 60 \times 21 \mathrm{~mm}$

Frequency stability: $\pm 2 \mathrm{Kh}$

\section{Mx2000 Receiver}

Weight: $125 \mathrm{~g}$

Size: $103 \times 60 \times 21 \mathrm{~mm}$

Battery required: $\mathrm{MN} 1604$

Sensitivity: $-98 \mathrm{dBm}$ for 40 -dB SINAD

\section{Headphones Specifications}

Sony, Dynamic stereo headphones, MDR-7506 Professional

\section{Video Camera Specifications}

Sony, CCD-TRV 65E HI8 